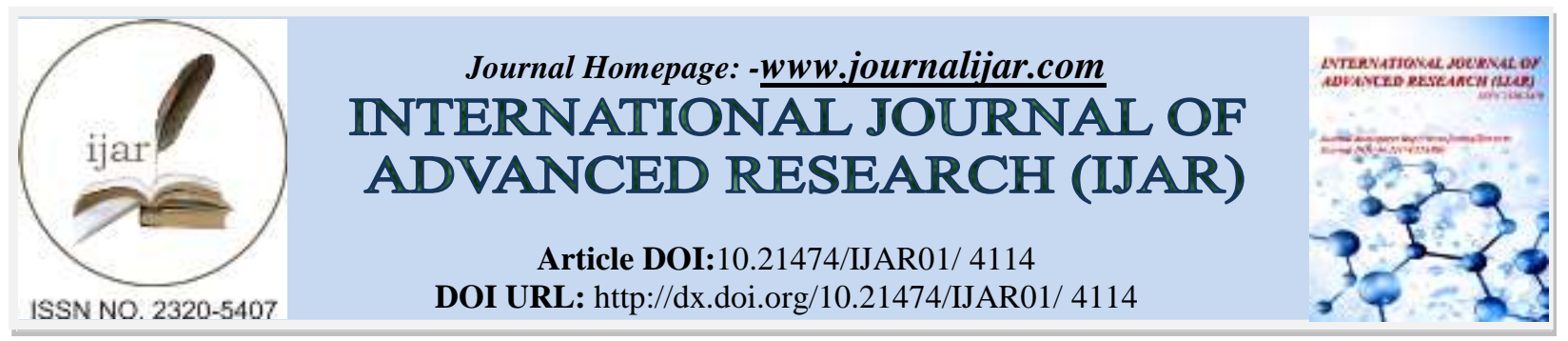

RESEARCH ARTICLE

\title{
ESTABLISHMENT OF A DIAGNOSTIC REFERENCE LEVEL FOR BRAIN CT PROCEDURES IN MOROCCAN HOSPITALS.
}

\section{Slimane Semghouli ${ }^{1, *}$, Bouchra Amaoui ${ }^{2}$, Abdennsser El Kharras ${ }^{3}$, Khalid Bouyakhlef ${ }^{4}$, Oum Keltoum Hakam $^{5}$ and Abdelmajid Choukri ${ }^{5}$.}

1. Department of Health Techniques, Higher Institute of Nursing Professions and Health Techniques, Agadir, Morocco.

2. Department of Radiotherapy, Regional Center of Oncology, Agadir, Morocco.

3. Department of Medical Imaging, Center of Medico-Surgical, Agadir. Morocco.

4. Laboratory of Biophysics, Faculty of Medicine and Pharmacy, Mohammed V University, Rabat, Morocco.

5. Department of Physics, Nuclear Physics and Techniques Research Unit, University of IbnTofail, Kenitra, Morocco.

\section{Manuscript Info}

Manuscript History

Received: 08 March 2017

Final Accepted: 0 April 2017

Published: May 2017

Key words:-

CT examinations, radiation Risks, effective Dose, DRLs.

\section{Abstract}

Purpose: The aim of this study is to evaluate the patient doses during Computed Tomography (CT) brain procedures in order to establish a local diagnostic reference level (DRL) in some hospitals in Morocco. Materials and methods: This study consisted of examining a total of 146 patients in six radiology departments in Morocco. The data were collected from CT Brain examinations at least of $20 \mathrm{CT}$ scans per hospital. For each examination reported, CT acquisition parameters have included number of series, use of contrast medium, tube $\mathrm{kV}$, tube current and rotation time, slice thickness, the displayed CT dose index $\left(\mathrm{CTDI}_{\mathrm{vol}}\right)$ and the Dose Length Product (DLP).

Results: The patients were examined with their own department protocol using multislice CT (MSCT) quadruple slice, 16 and 64 CT slice from different manufacturers. The tube current time produce is used in two hospitals (H4-AGA-CNSS, H5-RAB-INO). The patients are exposed to a high dose up 200 mAs. One hospital uses a higher value up to $130 \mathrm{kV}$ per CT brain. The tube tension defines penetration strength of the X ray beam. In terms of DLP, the average dose varies between 379,4 mGy.cm and $2434 \mathrm{mGy} . \mathrm{cm}$. Therefore, a third quartile value of 1408 mGy.cm can be used as a first DRL local for CT brain procedure for adults. The DRL value found is higher than that found in other countries. It is $14 \%$ to $42 \%$ higher compared to other studies. This value is as a preliminary study result, initiated to raise the attention concerning the prevention of unnecessary radiation dose for patients in CT imaging.

Conclusion: Doses received by patient during CT procedures vary from a radiology service to another and even at the same service. This large variation is due to patient clinical indication, CT system modality and image acquisition parameters.

The value of $1408 \mathrm{mGy} . \mathrm{cm}$ assessed as the first Moroccan DRL for CT brain procedure for adults is the highest value found so far in the 
literature. Therefore, in order to reduce this dose, corrective measures are required to eliminate unnecessary radiation dose that doesn't contribute to the overall profile of the patients. So, we recommend the normalisation of practices in the radiology departments by the development of such protocols and the promotion of educational and training programs in the field of radioprotection of patients.

Copy Right, IJAR, 2016,. All rights reserved.

\section{Introduction:-}

The diagnosis of a wide range of diseases by the introduction of CT is considered as a valuable medical imaging technique. Several new clinical applications continue to emerge in medical field after the development of powerful CT machines. In the last decade, the number of CT machines have increased and hence the CT examinations [1]. While the protection of patient from unnecessary radiation exposure is recommended, the patient's benefit from an accurate diagnosis must be greater than the risk of radiation exposure [2]. When the image acquisition parameters are not properly attuned according to the patient size, an unproductive radiation exposure may be delivered to the patient [3].Therefore, the reduction of the unnecessary exposure requires the application of the international commission of radiological protection (ICRP) principles of radiation protection. These principles specified that any medical imaging examination that necessitates exposure to ionizing radiation must be justified on the base of benefit to the patient. No practice requiring exposure to ionizing radiation must occur except if it generates adequate benefit to the exposed individual [4]. Once the radiological exam is justified, the technologist should use an optimum radiation exposure to accomplish the diagnostic task coherent with the requisite image quality. In order to evaluate the radiological practice, the ICRP recommended the establishment of diagnostic reference level (DRL) as the patient has no dose limit [5]. Since it first introduction in 1991, the establishment and application of DRL have formerly demonstrated beneficial tool for dose reduction in medical imaging procedure in order to reduce avoidable radiation exposure [6,7].In Morocco, which classified among countries with health care level III [8], there are about 100 CT machines with various modalities and manufacturers listed from a single slice to 128 slices. At the centre of optimization is the establishment of DRLs, earliest proposed by the ICRP in 1996 [5] and later introduced into European legislation [6]. DRLs permit the identification of anomalously high dose levels by tuning an upper threshold, which standard dose levels should not overtake when adequate practice is applied. Immoderate doses in $\mathrm{CT}$ are not as easily identified through image quality affects, as in norm film-based radiography. Therefore, a consciousness of typical dose levels permits CT users to rapidly identify and address any protocols which do not meet the ALARA principle (as low as reasonably achievable), as a result ameliorate radiographic exercise.

The rare studies on medical radiation exposure in Morocco $[9,10]$ and the lack of a previous study on the assessment of the absorbed dose for various scans and examinations in computed tomography $[11,12,13]$ prompted us to undertake this study.The purpose of this study is to evaluate the patient doses during CT brain procedures in order to establish a local diagnostic reference level DRL in some hospitals in Morocco.

\section{Materials and Methods:-}

The data used in this study were collected from six radiology services at various hospitals. Technical specifications of CT machines are regrouped in table 1 . The machines are regularly controlled for quality measurements and all the measure parameters were within acceptable range.

Table 1:- Scanner System.

\begin{tabular}{|c|l|l|c|}
\hline No & \multicolumn{1}{|c|}{ Hospital Reference } & \multicolumn{1}{|c|}{ Manufacture } & \multicolumn{1}{c|}{$\begin{array}{c}\text { Modality } \\
\text { (Number of Slice/detection) }\end{array}$} \\
\hline 1 & H1-OUJ-HF & GE LIGHT SPEED & 16 \\
\hline 2 & H2-RAB-CNRNS & GE LIGHT SPEED & 04 \\
\hline 3 & H3-AGA-HM & GE LIGHT SPEED & 04 \\
\hline 4 & H4-AGA-CNSS & GE LIGHT SPEED & 64 \\
\hline 5 & H5-RAB-INO & SEIMENS & 02 \\
\hline 6 & H6-AGA-HII & PHILIPS & \\
\hline
\end{tabular}




\section{Patient Data:-}

A total of 146 patients (76females' and70 males) referred for brain CT Imaging procedures were considered in this study in six different hospitals in Morocco. Patient related parameters (age, gender, diagnostic examination purpose, body region, use of contrast media) and patient dose were collected. In addition, other exposure related parameters were taken in consideration such gantry tilt, tension $(\mathrm{kV})$, tube current $(\mathrm{mA})$, exposure time, slice thickness, table increment, number of slices, and start and end positions of scans on patient dose the displayed CT dose index $\left(\mathrm{CTDI}_{\mathrm{vol}}\right)$ and the dose length product (DLP).

\section{Statistical Analysis:-}

The data were analyzed using Statistical Package for the Microsoft Office Excel 2007. DLP (mGy.cm) and CTDI ${ }_{\mathrm{vol}}$ (mGy) were analysed to obtain the third quartile value as a reference value for DRL for each hospital and the overall average.

\section{Results:-}

A total of 146 CT brain procedures were performed during the data collection period in six different hospitals. Patient age per hospital is presented in table 2 .

\begin{tabular}{|c|l|c|c|c|c|}
\hline \multicolumn{1}{|c|}{ Table 2: patient age per hospital } \\
\hline Variable & Hospital Reference & Average age(Y) & Std. Deviation & Maximum & Minimum \\
\hline \multirow{5}{*}{ Age } & H1-OUJ-HF & 58,15 & 08,75 & 82 & 43 \\
\cline { 2 - 6 } & H2-RAB-CNRNS & 51,50 & 21,00 & 90 & 17 \\
\cline { 2 - 6 } & H3-AGA-HM & 40,80 & 18,45 & 75 & 18 \\
\cline { 2 - 6 } & H4-AGA-CNSS & 46,44 & 19,63 & 77 & 22 \\
\cline { 2 - 6 } & H5-RAB-INO & 52,25 & 08,68 & 66 & 41 \\
\cline { 2 - 6 } & H6-AGA-HII & 44,62 & 18,64 & 80 & 21 \\
\hline
\end{tabular}

Table 3 and 4 present the tube current time product (mAs) and radiation exposure parameters for tube voltage ( $\mathrm{k} \mathrm{p}$ ), respectively.

Table 3:- Tube current time (mAs) product per hospital.

\begin{tabular}{|c|l|c|c|c|c|}
\hline Variable & Hospital Reference & $\begin{array}{c}\text { Mean of tube } \\
\text { current time }\end{array}$ & Std. Deviation & Maximum & Minimum \\
\hline \multirow{3}{*}{$\begin{array}{c}\text { Tube } \\
\text { current } \\
\text { time (mAs). }\end{array}$} & H1-OUJ-HF & 164,25 & 33,96 & 215,00 & 115,00 \\
\cline { 2 - 6 } & H2-RAB-CNRNS & 135,17 & 18,62 & 151,67 & 46,67 \\
\cline { 2 - 6 } & H3-AGA-HM & 151,65 & 58,54 & 315,00 & 70,00 \\
\cline { 2 - 6 } & H4-AGA-CNSS & 209,65 & 50,29 & 300,00 & 142,00 \\
\cline { 2 - 6 } & H5-RAB-INO & 320,00 & 00,00 & 320,00 & 320,00 \\
\cline { 2 - 6 } & H6-AGA-HII & 131,25 & 25,00 & 150,00 & 100,00 \\
\hline
\end{tabular}

Table 4:- Patient tube voltage $(\mathrm{kVp})$ product per hospital.

\begin{tabular}{|l|l|c|c|c|c|}
\hline Variable & Hospital Reference & Mean & Std. Deviation & Maximum & Minimum \\
\hline \multirow{5}{*}{$\mathbf{k}$} & H1-OUJ-HF & 120 & 0,00 & 120 & 120 \\
\cline { 2 - 7 } & H2-RAB-CNRNS & 140 & 0,00 & 140 & 140 \\
\cline { 2 - 7 } & H3-AGA-HM & 118 & 6,15 & 120 & 100 \\
\cline { 2 - 7 } & H4-AGA-CNSS & 120 & 0,00 & 120 & 120 \\
\cline { 2 - 7 } & H5-RAB-INO & 120 & 0,00 & 120 & 120 \\
\cline { 2 - 7 } & H6-AGA-HII & 120 & 0,00 & 120 \\
\hline
\end{tabular}

The patient dose in terms of DLP (mGy.cm) and $\mathrm{CTDI}_{\mathrm{vol}}$ are summarized in Tables 5 and 6 respectively. 
Table 5:- Brain dose and DRL per hospital.

\begin{tabular}{|c|c|l|c|c|c|c|}
\hline \multirow{2}{*}{ Variable } & Hospital Reference & Mean & $\begin{array}{c}\text { Std. } \\
\text { Deviation }\end{array}$ & Maximum & Minimum & $\begin{array}{c}\mathbf{3}^{\text {rd }} \\
\text { quartile }\end{array}$ \\
\hline \multirow{4}{*}{$\begin{array}{c}\text { DLP } \\
\text { (mGy.cm) }\end{array}$} & H1-OUJ-HF & 715,50 & 178,73 & 1035 & 340 & 860,72 \\
\cline { 2 - 7 } & H2-RAB-CNRNS & 2434 & 719,82 & 2900 & 922 & 2602,96 \\
\cline { 2 - 7 } & H3-AGA-HM & 1135,76 & 607,88 & 3072 & 281 & 1305,29 \\
\cline { 2 - 7 } & H4-AGA-CNSS & 1211,49 & 186,98 & 1637,41 & 906,03 & 1294,30 \\
\cline { 2 - 7 } & H5-RAB-INO & 1854 & 178,59 & 2134 & 1584 & 2009,5 \\
\cline { 2 - 7 } & H6-AGA-HII & 339 & 56 & 450 & 267 & 379,4 \\
\hline
\end{tabular}

Table 6:- $\mathrm{CTDI}_{\mathrm{vol}}$ and DRL per procedure.

\begin{tabular}{|c|c|c|c|c|c|c|}
\hline \multicolumn{9}{|c|}{ Hospital } & Mean & Std. Deviation & Maximum & Minimum & 3 $^{\text {rd }}$ quartile \\
\hline Variable & H1-OUJ-HF & 19,16 & 7,74 & 32,56 & 7,10 & 23,66 \\
\hline \multirow{4}{*}{$\begin{array}{c}\text { CTDI } \\
(\mathbf{m G y})\end{array}$} & H2-RAB-CNRNS & 117,84 & 39,20 & 119 & 49,64 & 119 \\
\cline { 2 - 7 } & H3-AGA-HM & 49,28 & 11,69 & 60,14 & 28,28 & 57,19 \\
\cline { 2 - 7 } & H4-AGA-CNSS & 74,34 & 10,57 & 95 & 59 & 75 \\
\cline { 2 - 7 } & H5-RAB-INO & 59,87 & 00,00 & 59,87 & 59,87 & 59,87 \\
\cline { 2 - 7 } & H6-AGA-HII & 22,3 & 00,00 & 22,3 & 22,3 & 22,3 \\
\hline
\end{tabular}

\section{Discussions:-}

Since its emergence in 1971, the computed tomography scan has been the highest growing medical imaging system. It owns a short scanning time and a volumetric acquisition, so it allows the diagnosis of various diseases. To raise the benefit of the imaging procedure, it is obligatory to assess the parameters that affect CT dose for the patient.

In this study, a total of $6 \mathrm{CT}$ machines were used, as shown in Table $1.33 \%$ of the equipment is 64 slices and 04 slices each and $17 \%$ is 16 slices and double slice each.

The majority of patients are medium aged patients, except in the hospitals H1-OUJ-HF and H3-AGA-HM. It is important to note that there are a number of young patients with age range from 17 to 25 years. For this age group where patients are more sensitive than older groups, appropriate to long life expectancy.

With regard to CT imaging, there are number of patient characters and scan parameters that affect the dose and the quality of image. Several CT scan are controlled by users (e.g. $\mathrm{kV}, \mathrm{mAs}$, pitch).Other factors are characteristic to the scanner (e.g, detector efficiency, geometry).While others are intrinsic to patient (e.g., patient size, anatomy scanned).All these parameters are linked. A solid comprehension of how each parameter links to the others and involves both dose and image quality is fundamental for preserving the dose as low as reasonably achievable (ALARA). Consequently, an accurate assess of the factors affecting patient dose is obligatory.

Table 3 summarizes the tube current time used per hospital; it is well recognized that the radiation dose is relative to patient doses $\left(\mathrm{CTDI}_{\mathrm{vol}}\right)$ throughout the radiological procedures. According to Table 3, all hospitals used a different average tube current even if some hospitals have the same machine. Despite there is no substantial difference of the majority of people head using different mean tube current is not justified. This reality proof that patients may had been exposed to needless radiations. The high tube current time produce is used in two hospitals (H4-AGA-CNSS, H5-RAB-INO).The patients are exposed to a high dose up to $200 \mathrm{mAs}$. When all parameters rest constant, the dose is proportional to tube current time product.

The tube voltage per hospital is presented in Table 4. Four hospitals out of six used a constant tube tension of $120 \mathrm{kVp}$.One hospital use a higher value up to 130 per CT brain. Tube tension defines penetration strength of the $\mathrm{x}-$ ray beam. Consequently, higher energy x-rays have a higher probability than lower energy X-ray of traversing through the body and making signal at the detector. With all else being equal, upper $\mathrm{kV}$ will augment the ratio signal/ noise. For the similar scan parameters, varying the $\mathrm{kV}$ from 120 to135 increases the dose by about $33 \%[14,15]$.One dose is higher and more photons reaching the detectors permit a reduction in image noise, but the contrast of the tissues is also compromised[15]. 
This study shows great variation in the radiation dose to the patients as presented in Table 5. Usually these distinctions of doses are due to differences in acquisition parameters such as tube voltages, tube current, number of scan and repeated scans. In terms of DLP, the mean varies from 1230,50 mGy.cm to 1822,74 mGy.cm for 4 slice and 64 slice respectively. Table 5 and 6 shows large variation in patient dose among different hospitals and even in the same hospital. There may be raisons for these disagreements in clinical environment, of which the mainly significant causes for this diversity were due to clinical indication, CT scan modality and imaging protocol. This disagreement is larger if the practitioners are not well trained in CT imaging protocols and radiation dose optimisation aspects. These factors show substantially versus measures to present effective radiation protection. Consequently, it is essential to establish the least exposure doorstep that will deliver an adequate image quality in each application, suitably formulated in terms of clinical effectiveness.

Figure 1 shows a comparison of the patient's DRL for CT brain procedures between several countries. The value of DRL proposed in this study is higher by $14 \%$ to $42 \%$ for the values established in the other countries. This value is a result of preliminary study initiated to raise the attention concerning the prevention of unnecessary radiation dose for patients in CT imaging. The Figure 1 indicates that there is a significant variation in DRL in diverse countries, and even in the same country from time to time due to progress in imaging technique. This study should be expanded to contain other surveys. The disposable data can be a baseline for more studies regarding dose optimisation. Based on our knowledge, no values have been suggested to date for DLP during CT brain procedure. Consequently, a third quartile value of $1408 \mathrm{mGy} . \mathrm{cm}$ can be used as a first DRL local for CT brain procedure for adults.

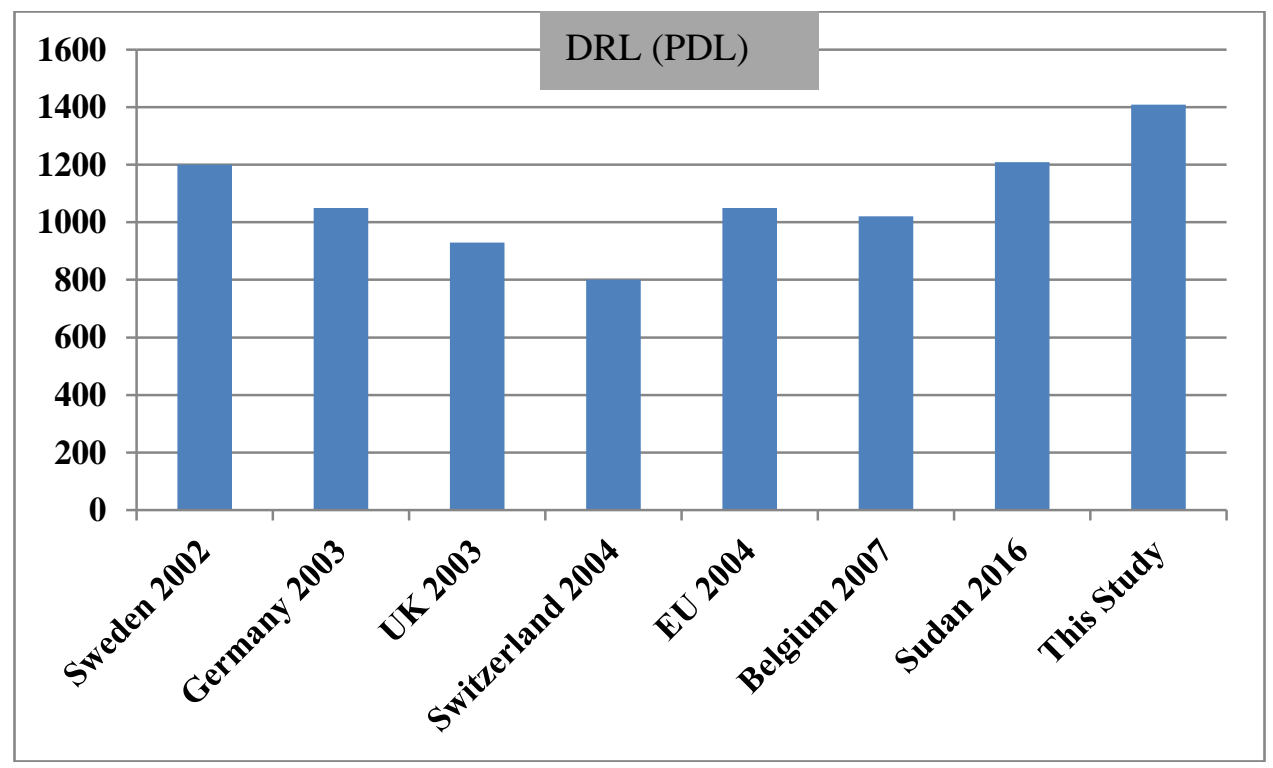

Figure 1:-Comparison of the DRL obtained in this study with other countries.

\section{Conclusion:-}

The purpose of this study was to evaluate the patient doses during CT brain procedures in order to establish a local diagnostic reference level DRL in six hospitals, therefore to deduct the first national DRL. This later assessed was to $1408 \mathrm{mGy} . \mathrm{cm}$ as the first Moroccan DRL for CT brain procedure for adults is higher to the equivalent result published for other countries. The high dose received by the patients in this study is attributed to the variation in technical parameters, clinical procedures, radiographic technique, untimely quality control program and perhaps the condition of the CT machine. Therefore, in order to reduce this dose, corrective measures are required in elimination unnecessary radiation that does not contribute to overall profile of the patients. Thus, we propose the unification of practices in our radiology departments by the development of the Moroccan protocols and investment in training in the field of patient radioprotection for our clinicians. 


\section{References:-}

1. Pearce MS, SalottiJA, Little MP, McHugh K., Lee C, Kim KP, Howe NL, Ronckers CM, Rajaraman P, Sir Craft AW, Parker L, Berrington de GonzàlezA, et al. Radiation exposure from CT scans in childhood and subsequent risk of leukaemia and brain tumours: a retrospective cohort study. Lancet. 2012;380:499-505.

2. Gregory KJ1, Bibbo G, Pattison JE. Uncertainties in effective dose estimates of adult CT head scans: the eect of head size. Med Phys. 2009 Sep;36(9):4121-5.

3. International Commission on Radiological Protection 1990 Recommendations of the International Commission on Radiological Protection. ICRP Publication 60. Annals. of the ICRP. 21 (1-3) : Pergamon Press, Oxford. (1991).

4. European Commission (EC). Radiation protection 109. Guidance on diagnostic reference levels (DRLs) for medical exposures. Directorate-General, Environment, Nuclear Safety and Civil Protection, 1999.

5. ICRP, Diagnostic reference levels in medical imaging: review and additional advice. Ann ICRP 2001,31 (4), 33.

6. Hart D, Wall B.F., “U.K. Population Dose From Medical X-ray Examinations,” European Journal of Radiology, June 2004.

7. Shrimpton P.C., Wall B.F., Hart D., "Diagnostic Medical Exposures in the U.K.," Applied Radiation and Isotopes, January 1999.

8. United Nations Scientic Committee on the Eects of Atomic Radiation (2000). 2000 Report to the General Assembly, Annex D Medical Radiation Exposures. United Nations, New York.

9. Semghouli S, Amaoui B, El Kharras A, Shaim, A, Choukri A and Hakam O. Physicians Knowledge of Radiation Risk in Prescribing CT Imaging in Moroccan Hospitals.British Journal of Applied Science \& Technology 20(3): 1-8, 2017; DOI: 10.9734/BJAST/2017/32491

10. Semghouli S, Amaoui B, El Fahssi M, Choukri A and O.M Hakam. Practitioners' Knowledge on Patients' Radioprotection in Emergency and Radiology Services of Hassan II Hospital Agadir Morocco. International Journal of Modern Physics and Applications. Volume. 1, No. 5, 2015, pp. 205-209. ISSN: 2381-6945 (Print); ISSN: 2381-6953 (Online).

11. Semghouli S, Amaoui B, Maamri A. Estimated radiation exposure from medical imaging for patients of radiology service of Al Faraby Hospital, Oujda Morocco. International Journal of Cancer Therapy and Oncology. Volume 3 • Number 3 • 2015. DOI: 10, 14319 / ijcto.33.25.

12. Aabid M, Semghouli S, Choukri A, Hakam O.K: Medical Radiation Exposure of Various Personnel Categories at National Center of Rehabilitation and Neurosciences (NCRNS) of Rabat. Scholars Journal of Applied Medical Sciences (SJAMS). Sch. J. App. Med. Sci., 2016; 4(8E):3071-3078.DOI: 10.21276/sjams.2016.4.8.66

13. Chahboub. Y, Choukri. A, Hakam. O.K, Talsmat. K, Benouhoud. M, andSemghouli. S (2016): Assessment of Absorbed Dose By Target Organs Using Mird Formalism During Certain Nuclear Medicine Procedures.European Journal of Biomedical and Pharmaceutical sciences. Volume 3, Issue 11, 61-64.

14. International Electrotechnical Commission (IEC). Medical electrical equipment. Part 2-44: Particular requirements for the safety of X-ray equipment or computed tomography. IEC 60601-2-44. Geneva, Switzerland; 1999.

15. Imaging Performance Assessments of CT (ImPACT). CT patient dosimetry spreadsheet (v0.99u, 10/12/2007). Retrieved April 2008.

16. Downes P, Jarvis R, Radu E, Kawrakow I, Spezi E. Monte Carlo simulation and patient dosimetry for a kilovoltage cone-beam CT unit. Med Phys. 2009;36(9):4156-4167.

17. Horiguchi J, Fujioka C, Kiguchi M, Yamamoto H, Kitagawa T, Kohno S, Ito K. Prospective ECG-triggered axial CT at $140-\mathrm{kV}$ tube voltage improves coronary in-stent restenosis visibility at a lower radiation dose compared with conventional retrospective ECG-gated helical CT. EurRadiol. 2009;19(10):2363-2372.

18. M.Elnour A, Youssef M, Sulieman A: Establishment of Local Diagnostic Reference Level For Brain CT Procedures. International Journal of Scientific Research. 2015 Volume : 4 Issue : 3 pp 295-298. 